\title{
Retrospective Study of The Corticosteroids Administration in Glioblastoma Patients as A Prognostic Factor in The Disease
}

\author{
Khaled Abdel Karim Mohamed, Mohamed Reda Kelany, Omar Mohamed Abdelrahman, \\ Abdelrahman Mostafa El-Sayed El-Adawy
}

Clinical Oncology and Nuclear Medicine Department, Faculty of Medicine, Ain Shams University
Corresponding author: Abdelrahman Mostafa El-Sayed El-Adawy, email: abdelrahmanadaway@ gmail.com

\begin{abstract}
Background: Glioblastoma multiforme (GBM) is the most common and most malignant of the glial tumors. Optimum management consists of maximal safe surgical resection, followed by concurrent chemoradiotherapy treatment then adjuvant chemotherapy with temozolomide. A considerable part from the care of patients with GBM involves general medical management, containing corticosteroids. Despite steroids common use, there is experimental and clinical evidence that corticosteroids have direct effects on tumor cell proliferation and apoptosis.
\end{abstract}

Aim of the Work: was to discuss the clinical relation between corticosteroids usage in Glioblastoma Multiforme (GBM) patients and quality of life as well as the disease progression free survival according to the recorded data from the joined hospitals.

Patients and Methods: Retrospective analysis of 66 adult patients diagnosed with GBM by surgery or imaging criteria. In order to assess the relation between corticosteroid dependence and the survival, patients were recruited into two groups (arms) according to dependency. Arm (A) was steroid dependent(34 patients) and arm (B) was steroid non-dependent (32 patients).

Results: Corticosteroids dependency was statistically significant correlated to both Overall Survival (OS)(median 2.5 in arm (A) vs. 13.1 months in the arm (B), p < 0.001), and Progression-free survival ratio (PFS)(median 2.3 in arm (A) vs. 9.4 months in arm (B), $p=0.035$ ). Also, steroid dependency was independent prognostic factor by doing the COX regression analysis.

Conclusion: Dependence on corticosteroids during course of treatment is identified as a poor prognostic factor.

Keywords: Corticosteroids, GBM, Glioblastoma multiforme, Dexamethasone, Prognostic Factor.

\section{INTRODUCTION}

According to Globocan web site, incidence of CNS tumors in Egypt was estimated by 6.6/100000. In a study that included 1618 cases of CNS tumors among Egyptians in delta region, Gliomas were of the highest frequency $35.2 \%$. Glioblastoma represented $38.3 \%$ of gliomas ${ }^{(1)}$.In another study performed in Ain Shams University Hospitals, a total number of registered newly diagnosed patients received during the study period were 943 cases. CNS tumors accounted for 53 newly diagnosed cases $(5.6 \%)$ and it was ranked as the fourth common cancer in both sexes. Glioblastoma Multiforme was the most common pathology occurring in about $43.3 \%$ of the presented cases ${ }^{(2)}$.

Exposure to ionising irradiation has been associated with increased risk of development of glioma, while association with the use of cell phones could not be confirmed in epidemiological studies. Rare hereditary syndromes carry an increased risk for glioma: Cowden-, Turcot-, Lynch-, Li-Fraumeni syndrome and neurofibromatosis type $1^{(3)}$.

GBM is the most aggressive diffuse glioma of astrocytic cell line and classified as grade IV in WHO Classification ${ }^{(4)}$. GBM remains an incurable disease with a median survival of 15 months ${ }^{(5)}$.
Treatment is complex and initially consists of maximal-safe surgical resection followed by radiation therapy (RT) with concurrent temozolomide (TMZ) chemotherapy followed by six cycles of maintenance $\mathrm{TMZ}^{(6)}$.

Steroids were introduced into the care of brain cancer patients nearly 60 years ago based on their powerful effects on tumor-induced edema. Ingraham pioneered the use of cortisone to treat postoperative cerebral edema in neurosurgical patients in 1952, and Kofman first used prednisone for peritumoral edema from brain metastases in 1957. More than 50 years ago, it was demonstrated that dexamethasone effectively alleviated cerebral edema due to brain tumors ${ }^{(7)}$.

Edema results from the flow of fluid into the extracellular space of the brain parenchyma through an incompetent blood-brain barrier (BBB) ${ }^{(8)}$.Dexamethasone is most commonly used by neuro-oncologists owing to its comparably minimal mineralocorticoid activity, possible lower risk of infection and occurrence of cognitive impairment. It has been suggested that corticosteroids produce their anti-edema effect by reducing the permeability of tumor capillaries ${ }^{(9)}$. 
Corticosteroids are commonly used perioperatively to control cerebral oedema and are frequently continued throughout subsequent treatment, especially during radiotherapy, to get control of its side effects. The effects of corticosteroids such as Dexamethasone (DEX) on cell growth in glioma models and on patient survival have remained controversial.

There is experimental and clinical evidence that corticosteroids have direct effects on tumor cell proliferation and apoptosis. Data from in vitro experiments suggest a variable inhibitory effect of dexamethasone on the proliferation of glioma cells. Dexamethasone and other glucocorticoid hormones have shown to decrease proliferation of embryonic neural stem cells with associated long-term effects on brain development. Dexamethasone, specifically through the glucocorticoid receptor, has an inhibitory effect on proliferation, but not differentiation of neural progenitor cells in the process of neurogenesis ${ }^{(10)}$.

In a recent study of Pitter et al they analyzed data from three large independent datasets which were further supported with a recent correlative retrospective analysis of 73 patients with glioblastoma by Shields et $\mathrm{al}^{(11)}$. Showing that DEX use during radiotherapy with concurrent TMZ correlated with reduced overall survival and progression-free survival. They couldn't exclude that detrimental effects of steroids other than direct interference with radiotherapy contributed to the overall inferior outcome of patients exposed to steroids. So steroids can contribute to morbidity and mortality through their direct toxicity, including steroid myopathy, impaired immune function, adrenal insufficiency, and bowel perforation ${ }^{(12)}$.

To support the direct detrimental effect of corticosteroids interfering with the activity of radiotherapy, Pitter et al.reported that pretreatment with DEX decreased the survival benefit offered by radiotherapy in murine gliomas. In these tumours, DEX decreased proliferation and the expression of many cell cycle-related genes. Expression of these genes inversely correlated with survival in The Cancer Genome Atlas (TCGA) glioblastoma patient dataset. Specifically, the p21 protein is induced by DEX in glioma cells, slows cell cycle progression and may confer cytoprotection ${ }^{(12)}$.
The aim of the current study was to discussthe clinical relation between corticosteroids usage in GBM patients and quality of life as well as the disease progression free survival according to the recorded data from the joined hospitals.

\section{PATIENTS AND METHODS}

The present retrospective descriptive analytical studyincluded 66 adult patients diagnosed as GBM by surgery or imaging criteria. They were recruited from Clinical Oncology and Nuclear Medicine Department, Faculty of Medicine - Ain Shams University, Department of Medical Oncology and Nuclear medicine, Egypt Air hospital and Hematology and Oncology hospital, Al Maadi Military Medical Compound. Date of data analysis was April 2017. The study was approved by the Ethics Board of Ain Shams University.

\section{Definition of survival}

Date of surgery was considered as the time of diagnosis. Progression free survival was calculated from the date of surgery to the date of disease progression at the primary location or other sites of the brain. Overall survival was calculated from the date of surgery to date of death due to any cause or to the date of data analysis.

Corticosteroids dependence is defined as the failure to withdraw the corticosteroids after initiating it as part of those patient treatment (with radiotherapy and TMZ) as relapse of the increased intra-cranial tension symptoms occur.

Non-dependence: is defined by the opposite of dependence, in which corticosteroids can be successfully withdrawn from the patients without recurrence of the ICT symptoms.

In order to assess the relation between corticosteroid dependence and the survival, patients were recruited into two groups (arms) according to dependency. Arm (A) steroid dependence (34 patients) and Arm (B) steroid non-dependence (32 patients).

Recorded toxicity was assessed by CTC version 4.0 according to patient's files.

\section{Statistical methods:}

Data were analyzed using statistical program for social science (SPSS) version 20. Quantitative data were expressed as mean \pm 
standard deviation (SD), median and range (minimum - maximum). Qualitative data were expressed as frequency and percentage.

\section{RESULTS}

\section{Patients' demographics:}

There were 21 women and 45 men, ranging in age from $25-75$ years, with a median age at diagnosis of 52.8 years. Patient performance status was recorded as measured by the ECOG score. Most common performance status was ECOG 1 as recorded in 26 patient of the study population. Only 14 patients had a medical record of fits in their disease course, see table 1.

\section{Disease characteristics:}

Primary site of the disease was divided according to lobe presentation into one lobe, bi-lobe and more than two lobes presentation; the most common presentation was bi-lobe presentation accounting for 36 patients about $54.5 \%$.

Biopsy was most common procedure done for the patients accounting for $57.6 \%$, while $15 \%$ of the patients were diagnosed by imaging criteria only.

\section{Progression and life status at the time of data collection:}

$63.6 \%$ of the patients died before progression was recorded, While $7.6 \%$ of the patients were alive at the time of the data collection. See table 1.

\section{Regarding treatment:}

$72.7 \%$ of the patients received radiotherapy as main treatment.59.1 \% of the whole cohort were treated by standard radiotherapy receiving a total dose of 60 Gy while, $13.6 \%$ were treated by hypo-fractionation radiotherapy with total dose up to $45 \mathrm{~Gy}$.

$62.1 \%$ of the patients administered Temozolomide concurrently with the radiotherapy. $51.5 \%$ of the patients continued Temozolomide as adjuvant protocol. Five patients received 2 nd line chemotherapy TMZ dose-intense was the most common line taken. Only one patient survived to receive other lines beyond 2 nd line. See table 1.
Table (1): Patient demographics, disease characteristics and treatment given:

\begin{tabular}{|c|c|c|c|}
\hline \multirow[t]{2}{*}{ Age (years) } & \multirow[t]{2}{*}{$\begin{array}{l}\text { Mean } \pm \text { SD } \\
\text { Range }\end{array}$} & \multicolumn{2}{|c|}{$\begin{array}{c}52.80 \pm 12.62 \\
25-75\end{array}$} \\
\hline & & No. $=66$ & Percentage \% \\
\hline \multirow{2}{*}{ Sex } & Female & 21 & $31.8 \%$ \\
\hline & Male & 45 & $68.2 \%$ \\
\hline \multirow{4}{*}{$\begin{array}{l}\text { Performance } \\
\text { status }\end{array}$} & 1 & 26 & $39.4 \%$ \\
\hline & 2 & 18 & $27.3 \%$ \\
\hline & 3 & 8 & $12.1 \%$ \\
\hline & 4 & 14 & $21.2 \%$ \\
\hline \multirow{2}{*}{ Fits } & No & 52 & $78.8 \%$ \\
\hline & Yes & 14 & $21.2 \%$ \\
\hline \multirow{3}{*}{ Site } & One Lobe & 24 & $36.4 \%$ \\
\hline & Bi Lobe & 36 & $54.5 \%$ \\
\hline & More than two & 6 & $9.1 \%$ \\
\hline \multirow{4}{*}{ Surgery } & None & 10 & $15.2 \%$ \\
\hline & Biopsy & 38 & $57.6 \%$ \\
\hline & Debulking & 8 & $12.1 \%$ \\
\hline & Maximal resection & 10 & $15.2 \%$ \\
\hline \multirow{2}{*}{ Progression } & No & 42 & $63.6 \%$ \\
\hline & Yes & 24 & $36.4 \%$ \\
\hline \multirow{2}{*}{ Life status } & Died & 61 & $92.4 \%$ \\
\hline & Alive & 5 & $7.6 \%$ \\
\hline \multirow{2}{*}{ Radiotherapy } & No & 18 & $27.3 \%$ \\
\hline & Yes & 48 & $72.7 \%$ \\
\hline \multirow{3}{*}{$\begin{array}{l}\text { Radiotherapy } \\
\text { doses }\end{array}$} & No & 18 & $27.3 \%$ \\
\hline & $45 \mathrm{GY}$ & 9 & $13.6 \%$ \\
\hline & $60 \mathrm{GY}$ & 39 & $59.1 \%$ \\
\hline \multirow{3}{*}{$\begin{array}{l}\text { Technique of } \\
\text { RTH }\end{array}$} & No & 18 & $27.3 \%$ \\
\hline & Hypo fraction & 9 & $13.6 \%$ \\
\hline & Standard & 39 & $59.1 \%$ \\
\hline \multirow{2}{*}{$\begin{array}{l}\text { Concomitant } \\
\text { TMZ }\end{array}$} & No & 25 & $37.9 \%$ \\
\hline & Yes & 41 & $62.1 \%$ \\
\hline \multirow{2}{*}{$\begin{array}{l}\text { Adjuvant } \\
\text { TMZ }\end{array}$} & No & 32 & $48.5 \%$ \\
\hline & Yes & 34 & $51.5 \%$ \\
\hline \multirow{2}{*}{ 2nd line } & No & 62 & $93.9 \%$ \\
\hline & Yes & 4 & $6.1 \%$ \\
\hline \multirow{6}{*}{$\begin{array}{l}\text { Type of } \\
\text { second line }\end{array}$} & No & 61 & $92.5 \%$ \\
\hline & TMZ dose-intense & 2 & $3 \%$ \\
\hline & therapy & 2 & \\
\hline & TMZ + Bev. & 1 & $1.5 \%$ \\
\hline & CCNU + Bev. & 1 & $1.5 \%$ \\
\hline & CVP & 1 & $1.5 \%$ \\
\hline \multirow{2}{*}{$\begin{array}{l}\text { Beyond 2nd } \\
\text { line }\end{array}$} & No & 65 & $98.5 \%$ \\
\hline & Yes & 1 & $1.5 \%$ \\
\hline
\end{tabular}

In order to assess the corticosteroids administration as a prognostic factor in disease progression, patients were recruited into two arms According to the definition of the corticosteroids dependence, previously discussed, (A and B).

34 patients were steroid dependant and were assigned to Arm (A)

32 patients were steroid independent and were assigned to Arm (B). See table 2.

Table (2): Corticosteroids dependence.

\begin{tabular}{|l|l|c|c|}
\hline \multicolumn{2}{|c|}{} & No. & $\%$ \\
\hline \multirow{2}{*}{ Dependence } & Arm A (dependent) & 34 & $51.5 \%$ \\
\cline { 2 - 4 } & Arm B (non dependent) & 32 & $48.5 \%$ \\
\hline
\end{tabular}


Corticosteroids dependency was statistically significant correlated to both OS (median 2.5 in the corticosteroids dependant group vs. 13.1 months in the non-corticosteroids dependant group, $\mathrm{p}<0.001$ ), and PFS (median 2.3 in the corticosteroids dependant group vs. 9.4 months in the non-corticosteroid dependant group, $\mathrm{p}=0.035$ ). See table $3 \& 4$.

Table (3): Comparison between median PFS in $\operatorname{arm} \mathrm{A}$ and $\operatorname{arm} \mathrm{B}$.

\begin{tabular}{|l|c|c|c|c|c|c|}
\hline & \multicolumn{2}{|c|}{ PFS (months) } & \multicolumn{2}{c|}{ 95\% CI } & \multicolumn{2}{c|}{ Log rank test } \\
\cline { 2 - 7 } & Median & SE & Lower & Upper & X2 & P-value \\
\hline Arm A (dependent) & 2.3 & 0.163 & 1.145 & 3.528 & \multirow{2}{*}{5.244} & \multirow{2}{*}{0.022} \\
\hline Arm B (non dependent) & 9.4 & 1.306 & 6.441 & 11.559 & & \\
\hline
\end{tabular}

Table (4): Comparison median OS in arm A and $\operatorname{arm~B.}$

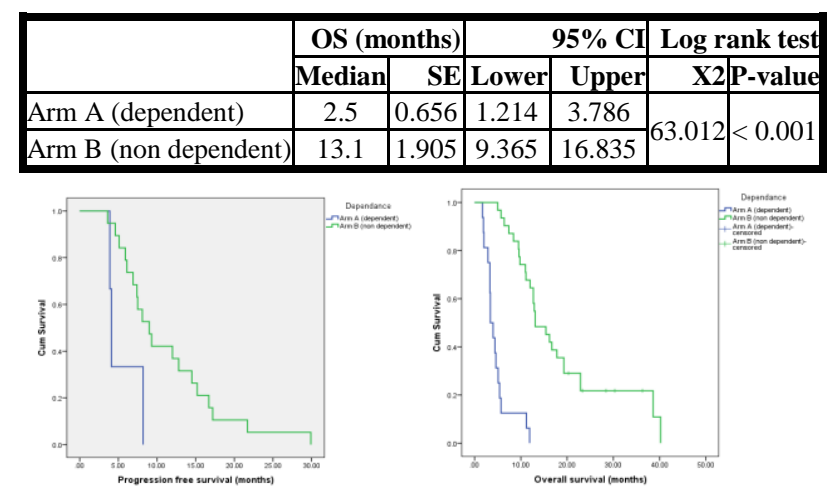

Figure (1): Kaplan-Mier curves for PFS and OS in Arm A and Arm B.

\section{DISCUSSION}

The corticosteroids are used to control neurological morbidity associated with brain tumors has been established as a standard of care decades ago. Except for primary CNS lymphoma where steroids are given as therapeutic agent owing to their direct cytotoxic effects, control of edema associated with brain tumors has been proposed to be the reason behind improvement of the disease symptoms ${ }^{(10)}$.

In support of that direct detrimental effect interfering with the activity of radiotherapy, Pitter et al. report that pretreatment with dexamethasone decreased the survival benefit afforded by radiotherapy in murine gliomas (glioma bearing mice). In these tumours, corticosteroids decreased proliferation and the expression of many cell cyclerelated genes. Expression of these genes inversely correlated with survival in the TCGA glioblastoma patient dataset. These genes are primarily known or predicted to be involved in proliferation, either via cell mitotic assembly, cycle checkpoints, DNA damage response and ATM signaling (12). Specifically, the p21 protein - cell cycle inhibitor is induced by corticosteroids in glioma cells, slows cell cycle progression and may confer cytoprotection.

In the current retrospective study, data were gathered from 66 patients with glioblastoma in whom corticosteroids administration was recorded. Corticosteroid dependency was more common in elder patients and those patients who presented with poor performance status. Patients with tumor of more than one lobe presentation were associated with corticosteroid dependency. Corticosteroids dependency was statistically significant correlated to both OS (median 4.1in the corticosteroids dependant group vs. 13.1 months in the non-corticosteroids dependant group, $\mathrm{p}<$ 0.001), and PFS (median 3.8 in the corticosteroids dependant group vs. 9.4 months in the noncorticosteroid dependant group, $\mathrm{p}=0.035)$. Also Multi-variate analysis was performed for all the factors affecting the survival in the study patients and we identified the administration of corticosteroids as poor prognostic factor for the disease outcome.

Our findings were further supported with a correlative retrospective analysis done by Shields et al.of 73 patients with glioblastoma showing that dexamethasone usage during radiotherapy with concurrent temozolomide was a poor prognostic indicator of both OS (median 12.7 vs. 22.5 months, $\mathrm{p}=0.02$ ), and PFS (median 6.0 vs. 8.8 months, $\mathrm{p}$ $=0.002)$ in the whole cohort ${ }^{(11)}$.

Another correlative result of a recent retrospective analysis done by $\mathrm{K}$. Pitter and his colleges $^{(12)}$ for a three large independent datasets, the first was 622 patients treated at Memorial Sloan Kettering Cancer Center (MSKCC) Patients not on DEX at the start of radiotherapy had a median survival of 20.6 months whereas patients on DEX had a survival time of 12.9 months $(\mathrm{P}>0.0001)$.

Second group, was 573 patients from the pivotal EORTC NCIC trial, Patients with baseline steroids had a lower median progression-free survival (5.3 versus 6.4, $\mathrm{P}>0.0001$ ) and a lower median overall survival (12 versus 17 months, P50.0001, HR = 1.56) and higher doses of steroids were a negative prognostic factor in patients treated 
with radiotherapy alone more than in patients treated with $\mathrm{TMZ} / \mathrm{RT} \rightarrow \mathrm{TMZ}^{(12)}$.

Third group was 832 glioblastoma patients enrolled in the German Glioma Network (GGN) the progression-free survival and overall survival were inferior in steroid-exposed patients in all patients pooled ${ }^{(12)}$.

They also did a Multi-variate analysis of the data in the three groups and they identified the use of corticosteroids early in the disease course, during radiotherapy without or with alkylating chemotherapy, as an independent predictor of poor outcome ${ }^{(12)}$.

These results might support the molecular background of determinate effect of steroid on radiotherapy of gliomas, but we should keep in our mind that others factors such as age and performance status of the patient are proved to be independent prognostic factors for both PFS and OS and those factors may indirectly co-related with steroid dependence so Further studies are warranted to more precisely establish interactions between corticosteroids and these cell types and how these might influence the disease course.

\section{CONCLUSION}

There is experimental and clinical evidence that corticosteroids have direct effects on tumor cell proliferation and apoptosis. Data from in vitro experiments suggest a variable, time dependent inhibitory effect of dexamethasone on the proliferation of glioma cells. Our results came consistent with the studies done recently; more usage of corticosteroids during course of treatment is a poor prognostic factor in the disease.

\section{REFERENCES}

1. Ferlay J (2015): Cancer incidence and mortality worldwide: sources, methods and major patterns in GLOBOCAN 2012.International journal of cancer, 136(5): 359-86.

2. Anwar W, Fahim H, Sos D et al. (2014): Establishment of computerized hospital-based cancer registry in the clinical oncology department of Ain shams university hospitals in Egypt: outlook on CNS tumors.abstract from Neuro-Oncology, 16(2): 72-72.

3. Alifieris $C$ and Trafalis $D$ (2015): Glioblastoma multiforme: Pathogenesis and treatment.Pharmacology \& Therapeutics, 152: 63-82.
4. Louis DN, Ohgaki H, Wiestler OD et al. (2007): The 2007 WHO classification of tumours of the central nervous system: Acta Neuropathologica, 114(2): 97-109.

5. Koshy M, Villano JL, Dolecek TA et al. (2012): Improved survival time trends for glioblastoma using the SEER 17 populationbased registries: Journal of Neuro-Oncology, 107(1): 207-212.

6. Stupp R, Mason WP, Van den Bent MJ et al. (2005): Radiotherapy plus concomitant and adjuvant temozolomide for glioblastoma: The New England journal of medicine, 352(10): 987-96.

7. Piette C, Munaut C, Foidart JM and Deprez $M$ (2006): Treating gliomas with glucocorticoids From bedside to bench: Acta Neuropathologica, 112(6): 651-664.

8. Wen PY, Schiff D, Kesari $S$ et al. (2006): Medical management of patients with brain tumors: Journal of Neuro-Oncology, 80(3): 313-332.

9. Hedley-Whyte ET and Hsu DW (1986): Effect of dexamethasone on blood-brain barrier in the normal mouse: Annals of neurology, 19(4): 373-7.

10. Dietrich J, Rao K, Pastorino $S$ and Kesari $S$ (2011): Corticosteroids in brain cancer patients: benefits and pitfalls: Expert Review of Clinical Pharmacology, 4(2), 233-242.

11. Shields L, Shelton BJ, Shearer AJ et al. (2015): Dexamethasone administration during definitive radiation and temozolomide renders a poor prognosis in a retrospective analysis of newly diagnosed glioblastoma patients: Radiation Oncology, 10(1), 4-11.

12. Pitter K L, Tamagno I, Alikhanyan $\mathrm{K}$ et al. (2016): Corticosteroids compromise survival in glioblastoma: Brain, 139(5), 1458-1471. 